\title{
Incidence and Pattern of Graft-versus-Host Disease in Patients Undergoing Allogeneic Transplantation after Nonmyeloablative Conditioning with Total Lymphoid Irradiation and Antithymocyte Globulin
}

\author{
Lauren Veltri, ${ }^{1}$ Michael Regier, ${ }^{2}$ Aaron Cumpston,,3 Sonia Leadmon,,3 William Tse, \\ Michael Craig, ${ }^{1,3}$ and Mehdi Hamadani ${ }^{1,3}$ \\ ${ }^{1}$ Osborn Hematopoietic Malignancy and Transplantation Program, West Virginia University, Morgantown, WV 26506, USA \\ ${ }^{2}$ Department of Biostatistics, West Virginia University, Morgantown, WV 26506, USA \\ ${ }^{3}$ Myeloma \& Lymphoma Service, West Virginia University, Morgantown, WV 26506, USA
}

Correspondence should be addressed to Mehdi Hamadani; mehdi.hamadani@gmail.com

Received 8 February 2013; Revised 29 March 2013; Accepted 30 March 2013

Academic Editor: Mark R. Litzow

Copyright (C) 2013 Lauren Veltri et al. This is an open access article distributed under the Creative Commons Attribution License, which permits unrestricted use, distribution, and reproduction in any medium, provided the original work is properly cited.

\begin{abstract}
Nonmyeloablative (NMA) conditioning with total lymphoid irradiation and antithymocyte globulin (TLI/ATG) has been shown to protect against acute graft-versus-host disease (GVHD). We report here our institutional experience with allogeneic transplantation following NMA conditioning with TLI/ATG $(n=21)$. GVHD prophylaxis consisted of a combination of a calcineurin inhibitor and mycophenolate mofetil. Median patient age was 59 years. The median followup of surviving patients is 545 days. One patient experienced primary graft rejection. The median time to neutrophil engraftment was 18 days and platelet engraftment was 9.5 days. The cumulative incidence (CI) of grade II-IV acute GVHD at day +100 was $28.6 \%$ and $38.1 \%$ at day +180 . The CI for grade III-IV acute GVHD was $28.6 \%$ at day +180 . CI of chronic GVHD was $45.2 \%$ at 1 year. The CI of disease relapse was $9.5 \%$ at 1 year. The rate of nonrelapse mortality (NRM) was $0 \%$ at day +100 and only $9.5 \%$ at 1 year. The overall and progression free survival at 1 year was $81 \%$ and $80.4 \%$, respectively. Our limited, retrospective data show encouraging relapse and NRM rates with TLI/ATG-based NMA conditioning, but with higher than previously reported rates of acute and chronic GVHD, underscoring the need for novel strategies designed to effectively prevent GVHD.
\end{abstract}

\section{Introduction}

Allogeneic hematopoietic cell transplantation (HCT) is a potentially curative modality for a variety of hematological malignancies $[1,2]$. However, the high rates of procedurerelated toxicities and nonrelapse mortality (NRM) have limited the applicability of HCT following conventional myeloablative conditioning regimens, to select cohort of younger patients with few or no comorbidities [3-8]. Hematological malignancies disproportionately affect the elderly [3]. From 2010 to 2030, the percentage of all cancers diagnosed in older adults in the United States will increase from $61 \%$ to $70 \%$ [9]. This change in demographics places an even greater emphasis on creating less toxic conditioning regimens, suitable for elderly or less fit individuals. The introduction of nonmyeloablative (NMA) and reduced-intensity conditioning (RIC) regimens has extended the use of allogeneic HCT to patients otherwise ineligible for conventional myeloablative HCT due to age or comorbidities $[3,10]$. However, even with the decline in transplant-related toxicities with the introduction of NMA regimens, graft-versus-host disease (GVHD) remains a major cause of morbidity and mortality [11-16].

NMA conditioning with total lymphoid irradiation (TLI) and antithymocyte globulin (ATG) has been shown in previous studies to protect against acute GVHD, while preserving graft-versus-malignancy (GVM) effects in both murine models and in the clinical realm [17-23]. NMA conditioning with TLI/ATG alters the host immune system to favor natural 
killer T cells, that suppress GVHD through polarization of donor conventional T cells towards secretion of noninflammatory cytokines (e.g., interleukin 4 ) and by promoting expansion of donor regulatory $\left(\mathrm{CD} 4^{+} \mathrm{CD} 25^{+} \mathrm{FoxP}^{+}\right) \mathrm{T}$ cells $[19,20,22]$. In the clinical setting, TLI and ATG-based conditioning has demonstrated low rates of NRM and acute GVHD $(<10 \%$ at day +100$)[17,19,21]$. Data on the incidence of late onset, classical acute GVHD in patients conditioned with TLI and ATG, beyond day +100 , are not available. We describe here our institutional experience with allogeneic HCT following NMA conditioning with TLI/ATG and report the incidence and pattern of early acute, late acute, and chronic GVHD.

\section{Patients and Methods}

2.1. Patient Population. Patients with hematological malignancies or bone marrow failure syndromes undergoing allogeneic HCT following NMA conditioning with TLI/ATG, between November 2007 and March 2012 at our Blood and Marrow Transplantation Program were included. The criteria used to offer TLI/ATG conditioning at our institution include presence of adverse-risk features (that preclude the use of higher-intensity conditioning regimens) defined by the presence of at least one of the following features: (i) age $\geq 60$-years; (ii) Karnofsky performance score (KPS) $\leq 70$; (iii) hematopoietic cell transplantation-comorbidity index (HCTCI) $\geq 4$ [24]; (iv) baseline diagnosis of follicular lymphoma, or chronic lymphocytic leukemia; and (v) prior history of autologous transplantation. This study was approved by the Institutional Review Board and Clinical Scientific Review Committee.

2.2. Conditioning Regimen. TLI/ATG conditioning regimen was delivered, as described previously [17, 19]. Briefly, neck, chest, abdomen, and pelvic computed tomography scans were obtained in all subjects and target volumes were outlined. The irradiation (i.e., clinical target volume) consisted of mantle field, a subdiaphragmatic field that included an inverted-Y, and splenic ports encompassing all major lymphoid organs (including the thymus, spleen, and lymph nodes) (Figure 1). Thoracic and abdominal organs at risk were contoured. TLI was administered from a $15 \mathrm{MeV}$ linear accelerator (photon beam), ten times in $80 \mathrm{cGy}$ fractions (total dose $800 \mathrm{cGy}$ ) on day 11 through day 7 and day 4 through day 1, using a 3D-conformal technique. One dose per day was given on days 11 through 7 , and days 4 through 2 . Two doses four hours apart were given on day 1 . Field junctions were required at the top of the spleen, and a second junction occasionally required, due to field size constraints, at the top of the pelvis. Isodose contours included the lymphoid regions and spleen at the $95 \%$ isodose levels. At field junctions, the $50 \%$ decrement lines of adjoining fields intersected at the midplane. Evenly weighted anterior-posterior and posterioranterior fields were treated using between 6 and $15 \mathrm{MV}$ photons from a linear accelerator. All fields were treated in each treatment session. ATG (Thymoglobulin, Genzyme, Cambridge, MA) was administered from day 11 through 7 at

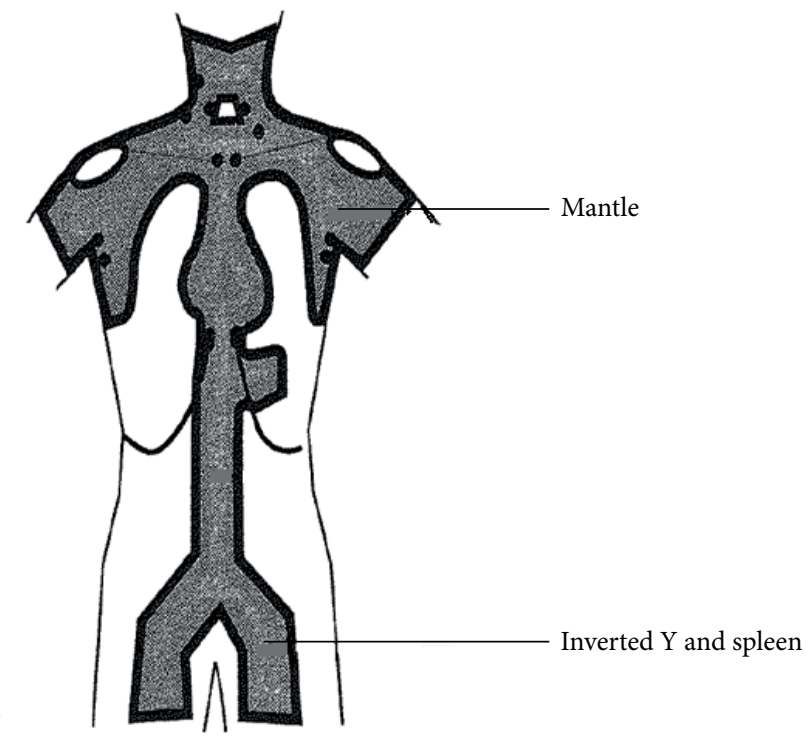

FIGURE 1: Lymph node regions included in total lymphoid irradiation.

the dose of $1.5 \mathrm{mg} / \mathrm{kg} /$ day. Hematopoietic stem cell infusion was performed on day 0 .

2.3. HLA Typing and Chimerism Analysis. High-resolution typing for human leukocyte antigen (HLA) class I (HLA-A,$\mathrm{B},-\mathrm{C})$ and class-II (HLA-DRB1, -DQB1) alleles was performed by polymerase chain reaction-sequence specific primary (PCR-SSP) amplification, as described previously [25]. To assess donor-cell chimerism, peripheral blood samples were collected before transplantation to identify PCR-short tandem repeat informative fragments for each donor/recipient pair. After transplantation chimerism analysis was performed on days $+30,+100,+180$, and +365 . Complete donor chimerism was defined as presence of $\geq 95 \%$ donor cells. Primary graft rejection was defined as failure to establish hematopoietic reconstitution of donor-origin after allografting, while secondary graft rejection was defined as confirmed loss of donor cells after initial donor-origin hematopoiesis. Secondary graft failure was defined as absolute neutrophil count (ANC) $<0.5 \times 10^{9} / \mathrm{L}$, after initial neutrophil recovery after HCT.

2.4. GVHD Prophylaxis. Patients undergoing matched sibling donor (MSD) HCT received GVHD prophylaxis with oral cyclosporine $(6.25 \mathrm{mg} / \mathrm{kg}$ twice a day; starting on day 3$)$ and mycophenolate mofetil (MMF; $15 \mathrm{mg} / \mathrm{kg}$ twice a day; starting on the evening of day 0 , after stem cell infusion). Patients undergoing an unrelated donor (URD) HCT received tacrolimus $(0.015 \mathrm{mg} / \mathrm{kg} / \mathrm{day}$ starting at day 3$)$ and MMF. Blood levels of cyclosporine and tacrolimus were monitored twice weekly. In the absence of acute GVHD or disease relapse, among recipients of MSD allografts, cyclosporine was tapered to discontinuation from day 56 to day 180, and MMF was stopped on day 28. Among recipients of URD transplants, 
tacrolimus was tapered to discontinuation between day 100 and day 180 , and MMF was tapered to discontinuation from day 42 to day 96 .

2.5. Transplantation Procedure and Supportive Care. All patients were treated in HEPA-filtered rooms and received fungal (fluconazole), herpes zoster/herpes simplex (acyclovir or valacyclovir), bacterial (ciprofloxacin or levofloxacin), and Pneumocystis jiroveci prophylaxis (trimethoprim/sulfamethoxazole or dapsone). Monitoring for cytomegalovirus (CMV) and Epstein-Barr virus (EBV) reactivation by quantitative PCR was conducted. Preemptive ganciclovir or valganciclovir were administered to patients with CMV reactivation (defined as $\geq 4000$ copies/mL, reconfirmed within 24 hours from initial detection); preemptive single intravenous dose of rituximab $\left(375 \mathrm{mg} / \mathrm{m}^{2}\right)$ was administered to patients with evidence of EBV replication (defined as $\geq 4000$ copies $/ \mathrm{mL}$, reconfirmed within 24 hours from initial detection). Urine and/or serum BK-virus PCR was obtained in all suspected cases of hemorrhagic cystitis. The time of neutrophil engraftment was considered the first of three successive days with ANC $\geq 0.5 \times 10^{9} / \mathrm{L}$ after posttransplantation nadir. The time of platelet engraftment was considered the first of seven consecutive days with platelet count $20 \times 10^{9} / \mathrm{L}$ or higher, in the absence of platelet transfusion for preceding seven days.

2.6. GVHD Assessment and Treatment. Patients achieving neutrophil engraftment were evaluable for acute GVHD. Acute GVHD was graded using standard criteria [26]. All potential cases of skin and gastrointestinal acute GVHD were confirmed on histological examination of representative biopsy specimens. Cases of liver only acute GVHD were also confirmed by either a transjugular or transcutaneous core needle biopsies. Patients were evaluable for chronic GVHD if engraftment occurred and the patient survived for 100 days after transplantation. The diagnoses of limited and extensive chronic GVHD were made as previously described [27-29], while classification of chronic GVHD into mild, moderate, and severe subtypes was performed by using the National Institutes of Health Consensus Development Project Criteria [30]. Corticosteroids comprised the first-line therapy of acute (grade II-IV) and extensive chronic GVHD. Second-line treatment was at the discretion of treating physicians.

2.7. Statistical Analysis. Descriptive statistics were calculated for the baseline variables of the whole cohort. Overall survival (OS) and progression free survival (PFS) were estimated using the Kaplan-Meier method. OS was defined as the time from transplant to death from any cause, and surviving patients were censored at last followup. PFS from transplantation was calculated using death and disease progression and/or relapse as events. NRM was defined as death from any cause other than disease progression or relapse. Cumulative incidence was estimated for NRM and relapse risk, with relapse as a competing risk for the former and death in remission for the latter [31]. Gray's test was used to assess the difference between various subgroups for NRM and relapse rate. The probability of developing acute GVHD or chronic
GVHD was depicted by calculating the cumulative incidence with relapse and death without relapse or acute GVHD or chronic GVHD as competing risks [14, 31]. Variables associated with acute and chronic GVHD, NRM, and relapse in the presence of a competing risk were tested individually using competing risk regression [32]. All $P$ values are two sided. Analyses were run using the statistical software $\mathrm{R}$ (http://www.r-project.org/).

\section{Results}

3.1. Patient Characteristics. The baseline characteristics of 21 patients included in this study are shown in Table 1 . The median age at baseline was 59 years (range 26-72), with nine patients $\geq 60$ years of age at transplantation. These patients received a median of 2 prior lines of therapies (range 1-6). At the time of transplantation 3 patients had chemotherapy refractory disease. Eight patients had an HCT-CI of $\geq 3$ at baseline. All except one patient received filgrastim-mobilized peripheral blood allografts. Five patients (23.8\%) underwent an MSD HCT. One patient undergoing an URD allogeneic transplant had a one allele-level mismatch at HLA-B with the donor. Twelve patients had KPS of $<90$ at baseline.

3.2. Engraftment and Chimerism. Twenty patients in the study successfully engrafted with donor cells. One patient experienced primary graft rejection and subsequently had autologous recovery (day +30 chimerism $=100 \%$ recipient cells). The median time to neutrophil engraftment was 18 days (range 9-82 days) and platelet engraftment was 9.5 days (range 6-24 days). Post HCT the ANC did not decrease below $500 / \mu \mathrm{L}$ in 8 patients. Four patients had secondary graft failure, which resolved with growth factor support. Median donor chimerism on days $+30,+100,+180$, and +365 was 92.5\% (range $0-100 \%$ ), 94\% (range $54-100 \%$ ), 100\% (range $56-100 \%$ ), and $100 \%$ (range $88-100 \%$ ), respectively. Donor lymphocyte infusions were administered in two patients (indication: decreasing donor chimerism and relapse acute myeloid leukemia).

3.3. GVHD. Twenty patients were evaluable for acute or chronic GVHD. The median time to tapering patients completely off immunosuppression was 218 days (range 78-458 days). Median time to onset of acute GVHD was 38 days (range 20-157). All acute GVHD cases were biopsy proven. The cumulative incidence of grade II-IV acute GVHD while accounting for competing events at day +100 and day +180 was $28.6 \%(95 \% \mathrm{CI}=1.2-62.9)(n=5)$ and $38.1 \%(95 \% \mathrm{CI}=$ 19.0-57.1) $(n=8)$, respectively (Figure $2(\mathrm{a}))$. The cumulative incidence for grade III-IV acute GVHD at day +100 and day +180 was $19 \%(95 \% \mathrm{CI}=0.1-69.1)(n=3)$ and $28.6 \%(95 \%$ $\mathrm{CI}=5.0-59.3)(n=5)$, respectively (Figure $2(\mathrm{~b}))$. The rate of acute GVHD was $20 \%(n=1)$ and $46.6 \%(n=7)$ in the recipient of MSD and URD transplants, respectively. When grouping the events by donor source (MSD versus URD), Gray's test indicated that there was no statistically significant difference between the two donor groups for grade II-IV acute GVHD $(P=0.429)$. 
TABLE 1: Baseline patient and disease characteristics.

\begin{tabular}{|c|c|}
\hline Characteristics & $N=21$ \\
\hline Median age; years (range) & $59(26-72)$ \\
\hline Male gender (\%) & $14(66.7)$ \\
\hline \multicolumn{2}{|l|}{ Diagnosis (\%) } \\
\hline Acute myeloid leukemia & 4 \\
\hline Chronic lymphocytic leukemia & 3 \\
\hline Chronic myeloid leukemia (blast crisis) & 2 \\
\hline B-cell NHL & 9 \\
\hline T-cell NHL & 2 \\
\hline Hemophagocytic syndrome & 1 \\
\hline \multicolumn{2}{|l|}{ Disease relapse risk ${ }^{*}(\%)$} \\
\hline Standard risk & $15(71.4 \%)$ \\
\hline High risk & $5(23.8 \%)$ \\
\hline Unknown & $1(4.8 \%)$ \\
\hline \multicolumn{2}{|l|}{ Prior autologous transplant (\%) } \\
\hline Yes & $1(4.8 \%)$ \\
\hline No & $20(95.2 \%)$ \\
\hline \multicolumn{2}{|l|}{ Donor type (\%) } \\
\hline Related donor & $5(23.8 \%)$ \\
\hline Unrelated donor & $16(76.2 \%)$ \\
\hline \multicolumn{2}{|l|}{ Sex mismatch $^{\dagger}(\%)$} \\
\hline $\mathrm{M} \rightarrow \mathrm{M}$ & $10(47.6 \%)$ \\
\hline $\mathrm{M} \rightarrow \mathrm{F}$ & $3(14.3 \%)$ \\
\hline $\mathrm{F} \rightarrow \mathrm{M}$ & $4(19.0 \%)$ \\
\hline $\mathrm{F} \rightarrow \mathrm{F}$ & $4(19.0 \%)$ \\
\hline \multicolumn{2}{|l|}{ Degree of HLA match (\%) } \\
\hline $10 / 10$ & $20(95.2 \%)$ \\
\hline $9 / 10$ & $1(4.8 \%)$ \\
\hline Median KPS (range) & $80(70-100)$ \\
\hline Median HCT-CI (range) & $1(0-5)$ \\
\hline \multicolumn{2}{|l|}{ Cytomegalovirus status (\%) } \\
\hline Patient or donor + & $11(52.4 \%)$ \\
\hline Both patient and donor + & $5(23.8 \%)$ \\
\hline Both patient and donor negative & $4(19.0 \%)$ \\
\hline Unknown & $1(4.8 \%)$ \\
\hline $\mathrm{ABO}$ mismatched & 8 \\
\hline $\begin{array}{l}\text { Median CD34 cell dose infused }\left(10^{6} \text { cells } / \mathrm{kg}\right. \\
\text { recipient) (range) }\end{array}$ & $6.0(1.5-11.3)$ \\
\hline \multicolumn{2}{|c|}{$\begin{array}{l}{ }^{\dagger} \text { Donor } \rightarrow \text { Patient. } \\
\text { Abbreviations: HCT-CI: hematopoietic cell transplantation comorbidity } \\
\text { index; KPS: Karnofsky performance score; NHL: non-Hodgkin lymphoma. } \\
\text { * Disease relapse-risk classification based on standard ASBMT criteria (avail- } \\
\text { able at http://www.asbmt.org/displaycommon.cfm?an=1\&subarticlenbr=35/ } \\
\text { (Last accessed February 1, 2013)). }\end{array}$} \\
\hline
\end{tabular}

Median time to the onset of chronic GVHD was 151 days (range 118-439). The cumulative of chronic GVHD was $45.2 \%(95 \% \mathrm{CI}=28.9-60.3)$ at 1 year (Figure 3$)$, while the cumulative incidence of limited and extensive chronic
GVHD was $18.6 \%(n=3)$ and $25.9 \%(n=5)$, respectively, at 1 year. The cumulative incidence of mild-moderate $(n=5)$ and severe $(n=3)$ chronic GVHD was $25.9 \%$ and $18.6 \%$, respectively at 1 year. When grouping the events by donor source, Gray's test indicated a weak statistical significance between the MSD and URD groups for chronic GVHD $(P=$ $0.188)$.

3.4. Infectious Complications. Viral reactivations were frequent. Fifteen patients (71.4\%) had CMV reactivation, while 2 experienced EBV reactivation. There were no cases of posttransplant lymphoproliferative disorder. Two patients had BK-virus associated episodes of hemorrhagic cystitis. Eleven patients $(52.4 \%)$ experienced bacterial infections in their posttransplant course. One patient developed an invasive fungal infection.

3.5. Nonrelapse Mortality and Relapse Rate. The cumulative of disease relapse was $9.5 \%(n=2)$ at 1 year. The cumulative incidence of NRM at day +100 and 1 year was $0 \%$ and $9.5 \%$, respectively (Figure 4 ). At last followup, five patients had died. Causes of death included disease relapse $(n=2)$, GVHD $(n=2)$, and motor vehicle accident $(n=1)$. When grouping the study population by donor source (MSD versus URD), Gray's test indicated that there was no statistically significant difference between the two donor groups for either NRM or relapse rates $(P=0.995$ and $P=0.418$ resp.).

3.6. Overall and Progression Free Survival. The median follow-up days of surviving patients are 545 days (range 1741664). At the last followup, 16 patients were alive. The 1-year and 3-year probabilities of OS were $81 \%$ and $72 \%$, respectively (Figure 5(a)). The respective figures for PFS are $80.4 \%$ and $70.4 \%$ (Figure 5(b)). There was no significant impact of donor source, patient age, or donor-cell chimerism on OS or PFS.

\section{Discussion}

Lower-intensity conditioning regimens (including the socalled RIC and NMA regimens) have extended the applicability of allogeneic HCT to elderly patients and those with comorbidities. In the current study, we report our institutional experience (extracted from a prospectively maintained database) with TLI/ATG-based NMA conditioning and make several interesting observations. First, despite including mostly elderly patients or those with significant comorbidities, TLI/ATG conditioning was associated with low NRM. Second, this truly NMA regimen was able to provide durable disease control, in this cohort of predominantly chemosensitive patients. Third, unlike prior reports, in our study, the rates of acute GVHD and chronic GVHD were high. Fourth, viral reactivations and infectious complications were frequent, but manageable.

In the updated experience from the Stanford group, the rates of grade II-IV acute GVHD at day +100 following TLI/ATG conditioning were $2 \%$ and $10 \%$ in patients receiving MSD and URD grafts, respectively [19]. Rates of late onset, classical acute GVHD were, however, not reported. 


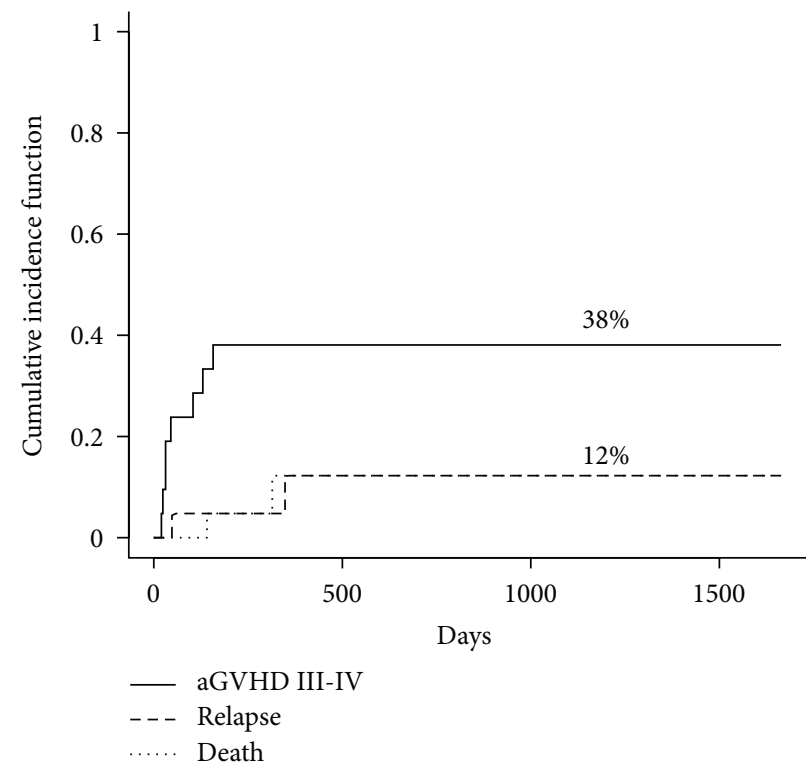

(a)

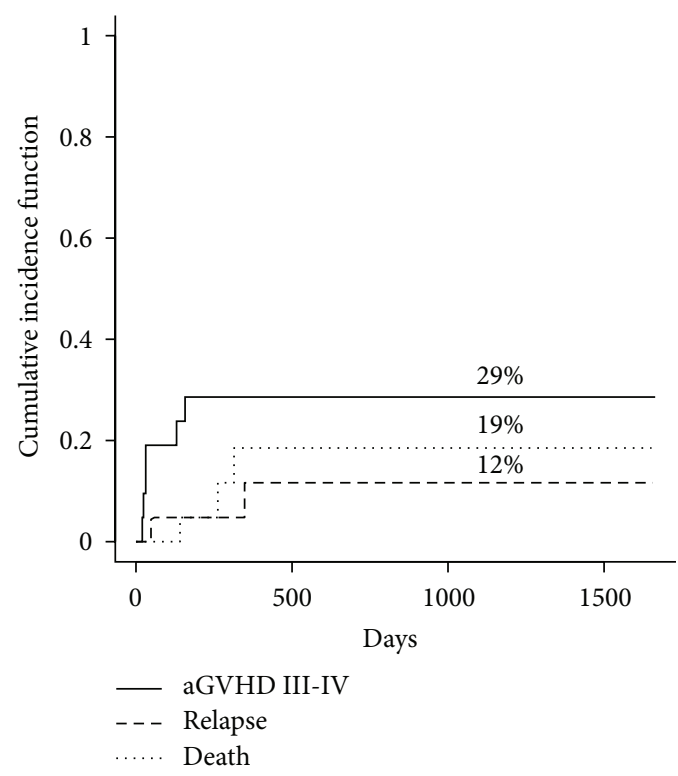

(b)

FIGURE 2: Cumulative incidence of grade II-IV (a) and grade III-IV (b) acute GVHD after transplantation (solid curves: acute GVHD, interrupted curves: competing events).

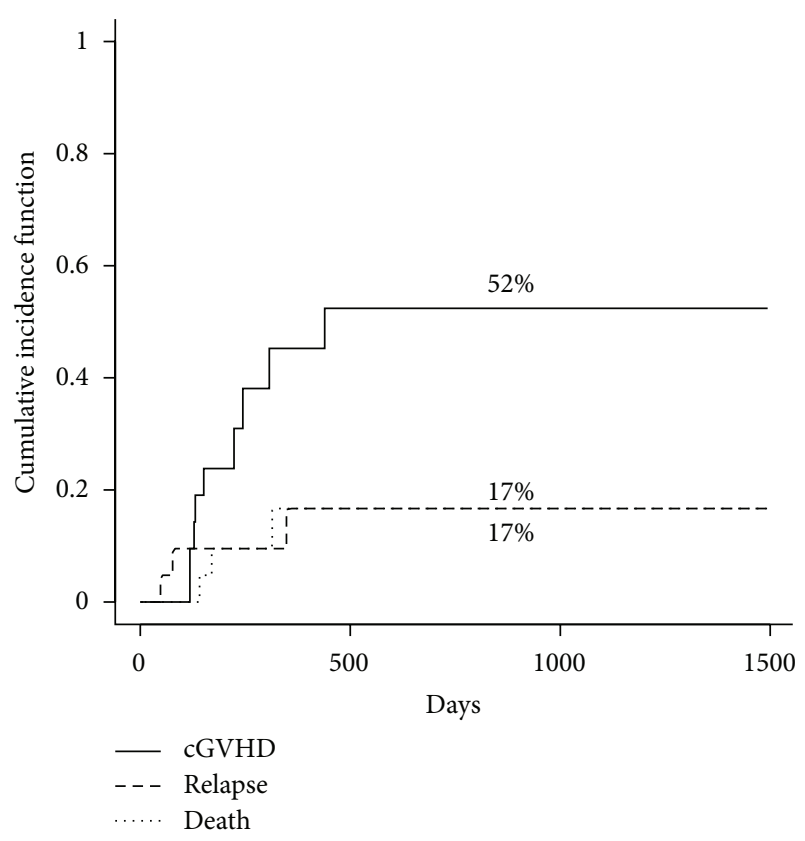

Figure 3: Cumulative incidence of chronic GVHD after transplantation (solid curves: chronic GVHD, interrupted curves: competing events).

Messina et al. in an Italian study reported acute GVHD rate of approximately $13 \%$ [21]. In contrast to these prior reports, our data demonstrate much higher rates of acute GVHD. Data regarding the incidence of late onset, classical acute GVHD following TLI/ATG conditioning are limited. Our study shows that late onset acute GVHD is not infrequent

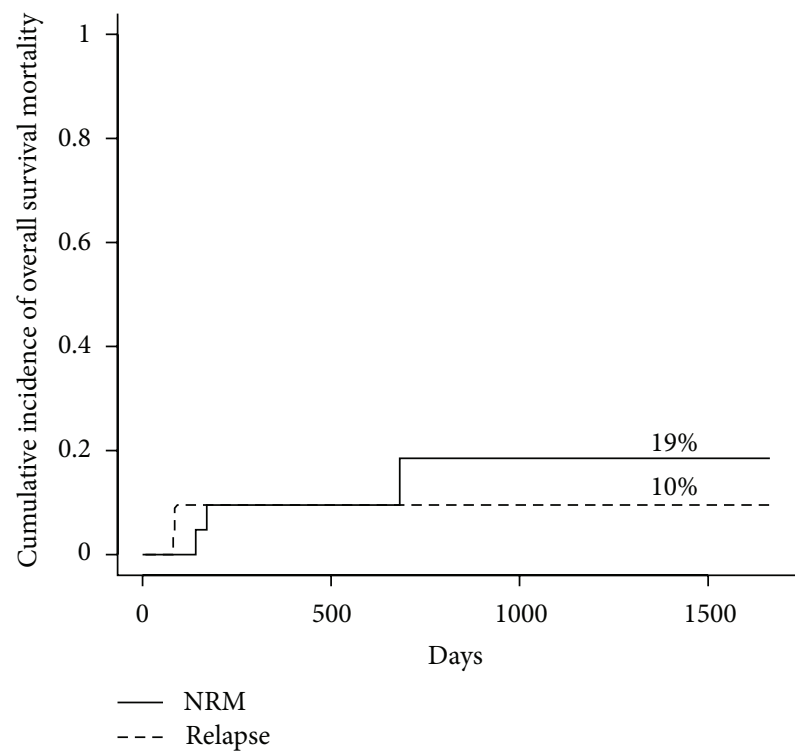

FIGURE 4: Cumulative incidence of NRM (solid curve) and disease relapse (interrupted curve), after transplantation.

after TLI/ATG, with cumulative incidence of acute GVHD increasing from $28.6 \%(n=5)$ at day +100 to $38.1 \%(n=8)$ at day +180 . The reasons for the higher rates of acute GVHD in our study compared to prior reports are not readily apparent. The proportion of URDs, HLA-mismatched recipients, and GVHD prophylaxis employed in our report is comparable to prior reports of TLI/ATG [17, 18, 21]. Interstudy variations in the observed incidence of acute GVHD have previously 


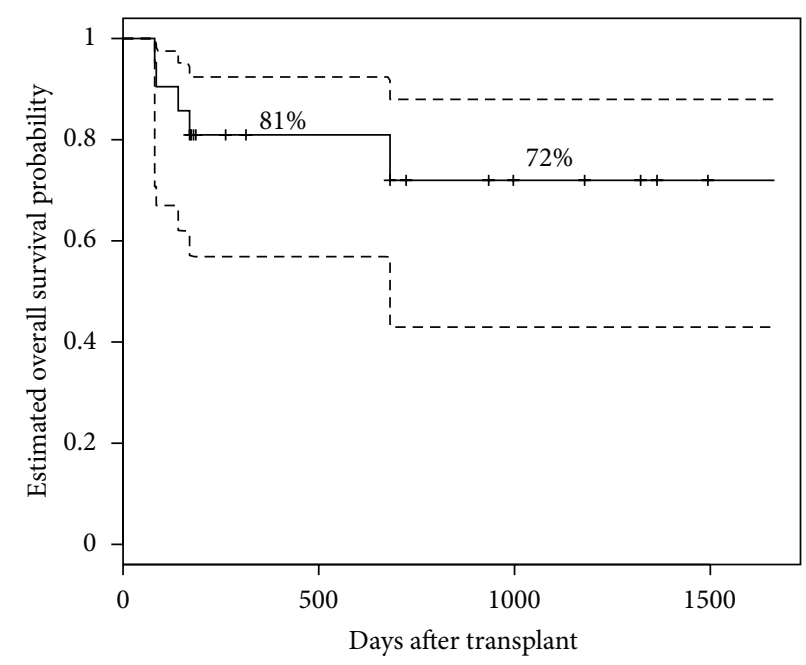

(a)

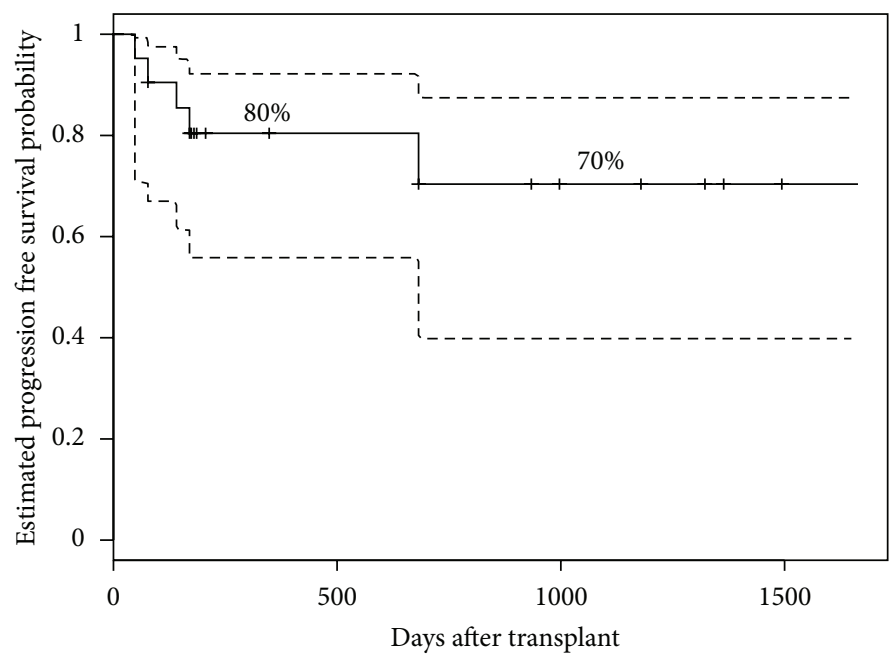

(b)

FIGURE 5: Kaplan-Meier estimates of overall survival (a) and progression free survival (b) after transplantation (interrupted curves = 95\% confidence intervals).

been shown by others to be a reflection of how aggressively diagnostic endoscopy is pursued to assess the etiology of gastrointestinal disturbances $[15,33]$. Our institutional practice of aggressively obtaining biopsy confirmation in all presentations suspicious for acute GVHD can be a reason for observed higher rates in our study. It is however important to note that all acute GVHD cases in our study had biopsy confirmation and that steroid refractory acute GVHD was the cause of death for two patients in our study, and a third patient who relapsed after transplant also had steroid refractory acute GVHD at the time of death. We substituted tacrolimus for cyclosporine in patients undergoing URD transplantation. This is unlikely to be responsible for our observed higher GVHD rates, as randomized data suggest improved efficacy of tacrolimus for GVHD prophylaxis compared to cyclosporine in URD transplantation [34]. We cannot discount the possibility that our data might merely be a reflection of a "center volume effect." The cumulative incidence of chronic GVHD ( $45.2 \%$ at 1 year) is also higher than previous TLI/ATG reports (26\%-35\%) [19, 21], but comparable to rates expected following non-TLI/ATG containing RIC or NMA regimens $[12,14,34,35]$.

The rate of CMV reactivation in our experience was high (71.4\%), likely due to ATG mediated T-cell depletion. In the report by Messina et al. [21], CMV reactivation was observed in $44 \%$ of the patients, while reactivation rates were not reported by Kohrt et al. [19]. The higher incidence of GVHD in our series and associated immunosuppressive therapy could have contributed to frequent CMV reactivations, as previously reported by others $[36,37]$. In fact, rate of CMV reactivation in our study in patients with acute GVHD (87.5\%; 7 of 8 patients with acute GVHD reactivated $\mathrm{CMV}$ ) was higher compared to the rate in patients without acute GVHD (61.5\%), albeit nonsignificantly $(P=0.20)$. The rates of CMV reactivation in patients with or without chronic GVHD in our study were $55 \%$ and $83 \%$, respectively
$(P=0.19)$. Despite the high acute GVHD rates in our study, the NRM and relapse rates remain reassuring. The rate of NRM in the Stanford experience was 3\% at 1 year, whereas the Italian study reported a 1 year NRM of $9.1 \%$ [19, 21]. Despite employing a truly NMA conditioning, the low rates of disease relapse in our study are noteworthy. It is possible that these low relapse rates are partially due to augmented GVM effects associated with development of GVHD or CMV reactivation, as reported recently [38]. One patient receiving a bone marrow product in our experience had primary graft rejection, raising the possibility that bone marrow might not be an optimal graft source for TLI/ATG based allogeneic HCT. It must however be noted that graft rejection rates of up to $5 \%$ with TLI/ATG have previously been reported $[18,19]$.

In conclusion, acknowledging the limitation of our study including its retrospective nature and small sample size, it appears that NMA conditioning with TLI/ATG provides durable disease control, with low rates of disease relapse and NRM. Clinically significant acute GVHD however remains frequent and problematic, underscoring the need for continued investigations to prevent this frequent cause of transplant morbidity and mortality.

\section{Conflict of Interests}

The authors declare that they have no conflict of interests.

\section{References}

[1] E. A. Copelan, "Hematopoietic stem-cell transplantation," The New England Journal of Medicine, vol. 354, no. 17, pp. 1813-1826, 2006.

[2] M. Hamadani, F. T. Awan, and E. A. Copelan, "Hematopoietic stem cell transplantation in adults with acute myeloid leukemia," Biology of Blood and Marrow Transplantation, vol. 14, no. 5, pp. 556-567, 2008. 
[3] M. L. Sorror, B. M. Sandmaier, B. E. Storer et al., "Long-term outcomes among older patients following nonmyeloablative conditioning and allogeneic hematopoietic cell transplantation for advanced hematologic malignancies," JAMA, vol. 306, no. 17, pp. 1874-1883, 2011.

[4] M. L. Sorror, B. E. Storer, D. G. Maloney, B. M. Sandmaier, P. J. Martin, and R. Storb, "Outcomes after allogeneic hematopoietic cell transplantation with nonmyeloablative or myeloablative conditioning regimens for treatment of lymphoma and chronic lymphocytic leukemia," Blood, vol. 111, no. 1, pp. 446-452, 2008.

[5] S. Slavin, A. Nagler, E. Naparstek et al., "Nonmyeloablative stem cell transplantation and cell therapy as an alternative to conventional bone marrow transplantation with lethal cytoreduction for the treatment of malignant and nonmalignant hematologic diseases," Blood, vol. 91, no. 3, pp. 756-763, 1998.

[6] H. G. Klingemann, R. Storb, and A. Fefer, "Bone marrow transplantation in patients aged 45 years and older," Blood, vol. 67, no. 3, pp. 770-776, 1986.

[7] P. A. McSweeney, D. Niederwieser, J. A. Shizuru et al., "Hematopoietic cell transplantation in older patients with hematologic malignancies: replacing high-dose cytotoxic therapy with graft-versus-tumor effects," Blood, vol. 97, no. 11, pp. 3390-3400, 2001.

[8] O. Ringden, M. M. Horowitz, R. P. Gale et al., "Outcome after allogeneic bone marrow transplant for leukemia in older adults," Journal of the American Medical Association, vol. 270, no. 1, pp. 57-60, 1993.

[9] B. D. Smith, G. L. Smith, A. Hurria, G. N. Hortobagyi, and T. A. Buchholz, "Future of cancer incidence in the United States: burdens upon an aging, changing nation," Journal of Clinical Oncology, vol. 27, no. 17, pp. 2758-2765, 2009.

[10] B. Gyurkocza, R. Storb, B. E. Storer et al., "Nonmyeloablative allogeneic hematopoietic cell transplantation in patients with acute myeloid leukemia," Journal of Clinical Oncology, vol. 28, no. 17, pp. 2859-2867, 2010.

[11] V. T. Ho and R. J. Soiffer, "The history and future of T-cell depletion as graft-versus-host disease prophylaxis for allogeneic hematopoietic stem cell transplantation," Blood, vol. 98, no. 12, pp. 3192-3204, 2001.

[12] M. Hamadani, W. Blum, G. Phillips et al., "Improved nonrelapse mortality and infection rate with lower dose of antithymocyte globulin in patients undergoing reduced-intensity conditioning allogeneic transplantation for hematologic malignancies," Biology of Blood and Marrow Transplantation, vol. 15, no. 11, pp. 1422-1430, 2009.

[13] M. Mielcarek, P. J. Martin, W. Leisenring et al., "Graftversus-host disease after nonmyeloablative versus conventional hematopoietic stem cell transplantation," Blood, vol. 102, no. 2, pp. 756-762, 2003.

[14] M. Mohty, J. O. Bay, C. Faucher et al., "Graft-versus-host disease following allogeneic transplantation from HLA-identical sibling with antithymocyte globulin-based reduced-intensity preparative regimen," Blood, vol. 102, no. 2, pp. 470-476, 2003.

[15] J. Pidala, J. Kim, H. Jim et al., "A randomized phase II study to evaluate tacrolimus in combination with sirolimus or methotrexate after allogeneic hematopoietic cell transplantation," Haematologica, vol. 97, no. 12, pp. 1882-1889, 2012.

[16] O. Sala-Torra, P. J. Martin, B. Storer et al., "Serious acute or chronic graft-versus-host disease after hematopoietic cell transplantation: a comparison of myeloablative and nonmyeloablative conditioning regimens," Bone Marrow Transplantation, vol. 41, no. 10, pp. 887-893, 2008.
[17] R. Lowsky, T. Takahashi, P. L. Yin et al., "Protective conditioning for acute graft-versus-host disease," The New England Journal of Medicine, vol. 353, no. 13, pp. 1321-1331, 2005.

[18] H. Kohrt and R. Lowsky, "Nonmyeloablative conditioning with total lymphoid irradiation and antithymocyte globulin: an update," Current Opinion in Hematology, vol. 16, no. 6, pp. 460465, 2009.

[19] H. E. Kohrt, B. B. Turnbull, K. Heydari et al., "TLI and ATG conditioning with low risk of graft-versus-host disease retains antitumor reactions after allogeneic hematopoietic cell transplantation from related and unrelated donors," Blood, vol. 114, no. 5, pp. 1099-1109, 2009.

[20] F. Lan, D. Zeng, M. Higuchi, J. P. Higgins, and S. Strober, "Host conditioning with total lymphoid irradiation and antithymocyte globulin prevents graft-versus-host disease: the role of CD1-reactive natural killer T cells," Biology of Blood and Marrow Transplantation, vol. 9, no. 6, pp. 355-363, 2003.

[21] G. Messina, L. Giaccone, M. Festuccia et al., "Multicenter experience using total lymphoid irradiation and antithymocyte globulin as conditioning for allografting in hematological malignancies," Biology of Blood and Marrow Transplantation, vol. 18, no. 10, pp. 1600-1607, 2012.

[22] A. B. Pillai, T. I. George, S. Dutt, and S. Strober, "Host natural killer $\mathrm{T}$ cells induce an interleukin-4-dependent expansion of donor $\mathrm{CD}^{+} \mathrm{CD}^{+} 5^{+}$Foxp $^{+} \mathrm{T}$ regulatory cells that protects against graft-versus-host disease," Blood, vol. 113, no. 18, pp. 4458-4467, 2009.

[23] S. Strober, "Protective conditioning against GVHD and graft rejection after combined organ and hematopoietic cell transplantation," Blood Cells, Molecules, and Diseases, vol. 40, no. 1, pp. 48-54, 2008.

[24] M. L. Sorror, M. B. Maris, R. Storb et al., "Hematopoietic cell transplantation (HCT)-specific comorbidity index: a new tool for risk assessment before allogeneic HCT," Blood, vol. 106, no. 8, pp. 2912-2919, 2005.

[25] N. Flomenberg, L. A. Baxter-Lowe, D. Confer et al., "Impact of HLA class I and class II high-resolution matching on outcomes of unrelated donor bone marrow transplantation: HLA-C mismatching is associated with a strong adverse effect on transplantation outcome," Blood, vol. 104, no. 7, pp. 19231930, 2004.

[26] D. Przepiorka, D. Weisdorf, P. Martin et al., "Consensus conference on acute GVHD grading," Bone Marrow Transplantation, vol. 15, no. 6, pp. 825-828, 1995.

[27] E. R. Farmer, “The histopathology of graft-versus-host disease," Advances in dermatology, vol. 1, pp. 173-188, 1986.

[28] H. M. Shulman, K. M. Sullivan, and P. L. Weiden, "Chronic graft-versus-host syndrome in man. A long-term clinicopathologic study of 20 seattle patients," American Journal of Medicine, vol. 69, no. 2, pp. 204-217, 1980.

[29] K. M. Sullivan, E. Agura, C. Anasetti et al., "Chronic graftversus-host disease and other late complications of bone marrow transplantation," Seminars in Hematology, vol. 28, no. 3, pp. 250-259, 1991.

[30] A. H. Filipovich, D. Weisdorf, S. Pavletic et al., "National institutes of health consensus development project on criteria for clinical trials in chronic graft-versus-host disease: I. diagnosis and staging working group report," Biology of Blood and Marrow Transplantation, vol. 11, no. 12, pp. 945-956, 2005. 
[31] T. A. Gooley, W. Leisenring, J. Crowley, and B. E. Storer, "Estimation of failure probabilities in the presence of competing risks: new representations of old estimators," Statistics in Medicine, vol. 18, no. 6, pp. 695-706, 1999.

[32] J. P. Fine and R. J. Gray, "A proportional hazards model for the subdistribution of a competing risk," Journal of the American Statistical Association, vol. 94, no. 446, pp. 496-509, 1999.

[33] P. J. Martin, G. B. McDonald, J. E. Sanders et al., "Increasingly frequent diagnosis of acute gastrointestinal graft-versus-host disease after allogeneic hematopoietic cell transplantation," Biology of Blood and Marrow Transplantation, vol. 10, no. 5, pp. 320-327, 2004.

[34] R. A. Nash, J. H. Antin, C. Karanes et al., "Phase 3 study comparing methotrexate and tacrolimus with methotrexate and cyclosporine for prophylaxis of acute graft-versus-host disease after marrow transplantation from unrelated donors," Blood, vol. 96, no. 6, pp. 2062-2068, 2000.

[35] J. Finke, W. A. Bethge, C. Schmoor et al., "Standard graft-versushost disease prophylaxis with or without anti-T-cell globulin in haematopoietic cell transplantation from matched unrelated donors: a randomised, open-label, multicentre phase 3 trial," The Lancet Oncology, vol. 10, no. 9, pp. 855-864, 2009.

[36] B. George, I. H. Kerridge, N. Gilroy et al., "A risk score for early cytomegalovirus reactivation after allogeneic stem cell transplantation identifies low-, intermediate-, and high-risk groups: reactivation risk is increased by graft-versus-host disease only in the intermediate-risk group," Transplant Infectious Disease, vol. 14, no. 2, pp. 141-148, 2012.

[37] P. Ljungman, C. Cordonnier, H. Einsele et al., "Use of intravenous immune globulin in addition to antiviral therapy in the treatment of CMV gastrointestinal disease in allogeneic bone marrow transplant patients: a report from the European group for blood and marrow transplantation (EBMT)," Bone Marrow Transplantation, vol. 21, no. 5, pp. 473-476, 1998.

[38] A. H. Elmaagacli, N. K. Steckel, M. Koldehoff et al., "Early human cytomegalovirus replication after transplantation is associated with a decreased relapse risk: evidence for a putative virus-versus-leukemia effect in acute myeloid leukemia patients," Blood, vol. 118, no. 5, pp. 1402-1412, 2011. 


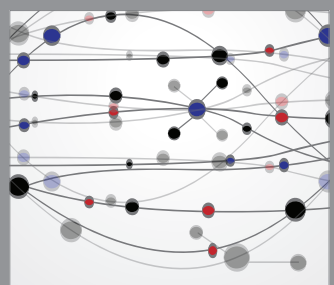

The Scientific World Journal
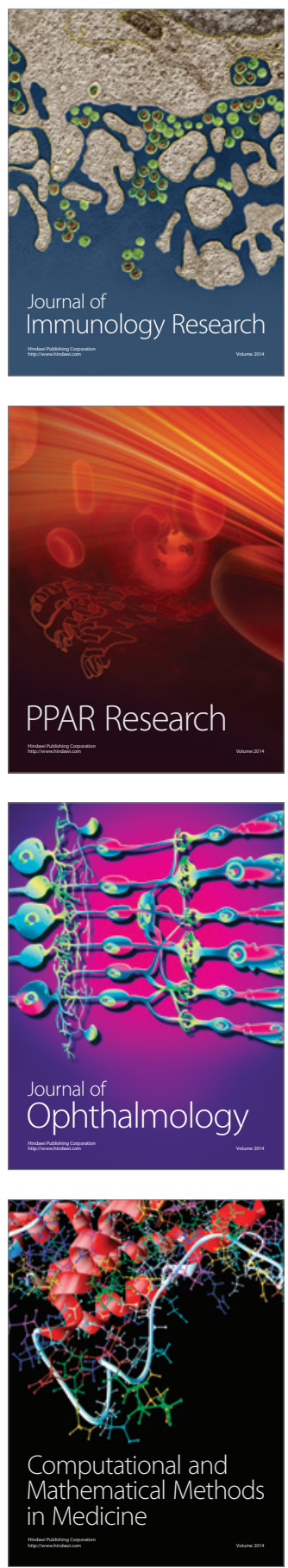

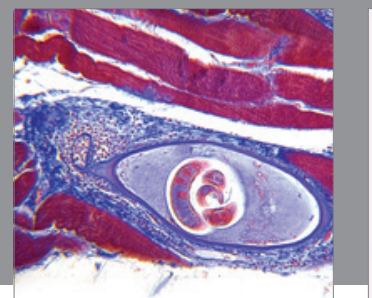

Gastroenterology

Research and Practice
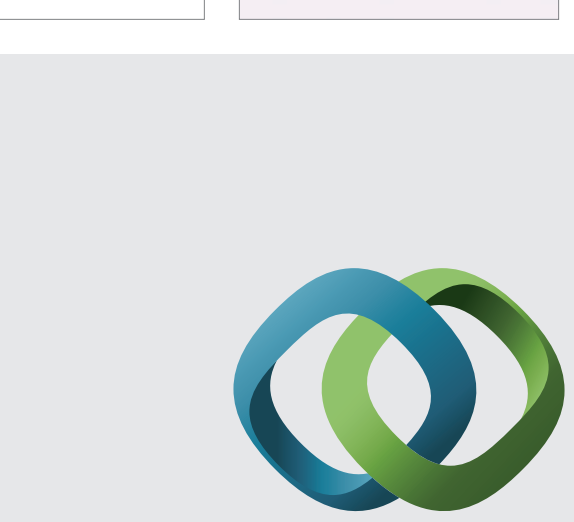

\section{Hindawi}

Submit your manuscripts at

http://www.hindawi.com
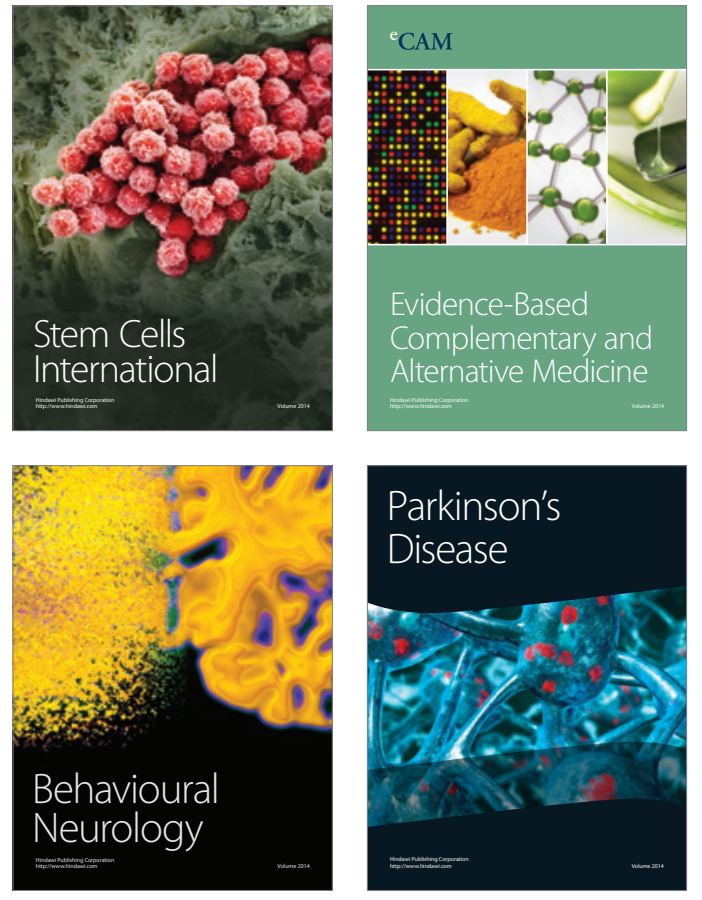
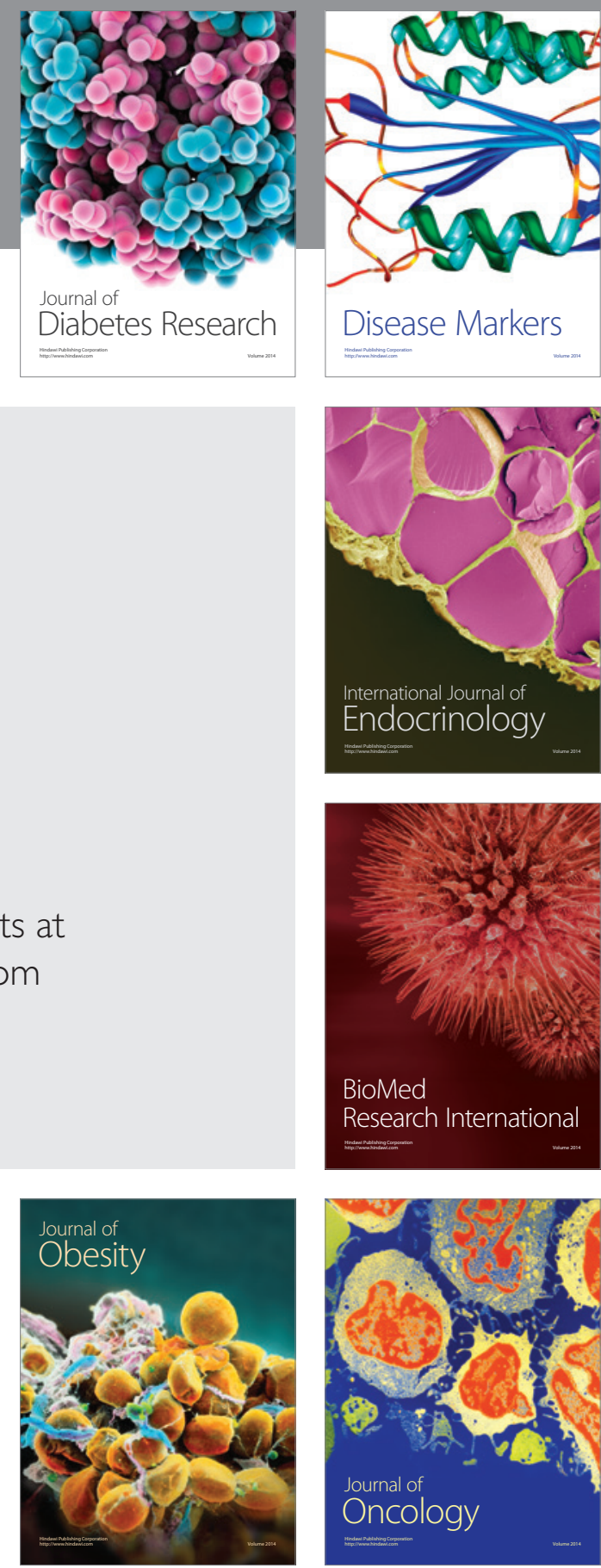

Disease Markers
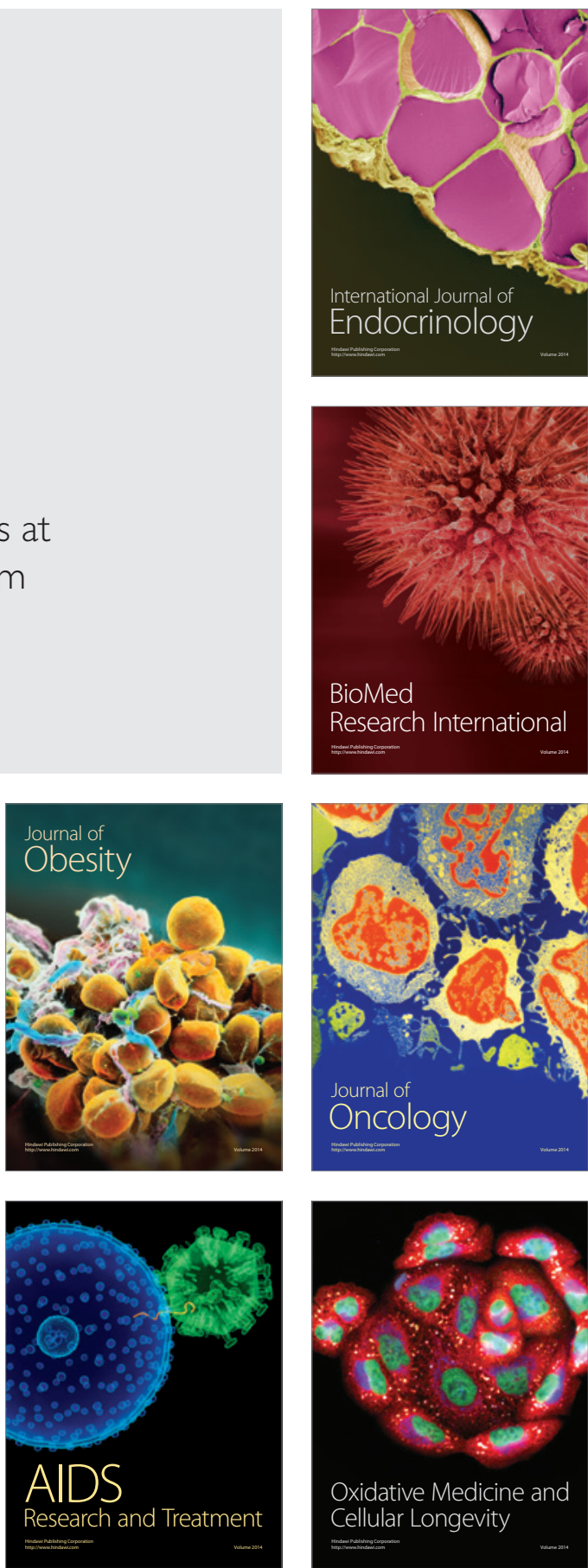